\title{
The Effects of Organic Solvents on the Physicochemical Properties of Human Serum Albumin Nanoparticles
}

\author{
Hossein Mohammad-Beigi ${ }^{1}$, Seyed Abbas Shojaosadati 1*, Dina Morshedi ${ }^{2}$, Negar Mirzazadeh 1, Ayyoob \\ Arpanaei ${ }^{2}$ \\ ${ }^{1}$ Biotechnology Group, Faculty of Chemical Engineering, Tarbiat Modares University, Tehran, Iran \\ ${ }^{2}$ Department of Industrial and Environmental Biotechnology, National Institute of Genetic Engineering and Biotechnology, \\ Tehran, Iran
}

\begin{abstract}
${ }^{*}$ Corresponding author: Seyed Abbas Shojaosadati, Department of Biotechnology Group, Faculty of Chemical Engineering, Tarbiat Modares University, Tehran, Iran. Tel: +98 21 82883341, Fax: +98-2182883381, E-mail: shoja_sa@modares.ac.ir
\end{abstract}

Received: February 21, 2015; Revised: August 28, 2015; Accepted: October 12, 2015

\begin{abstract}
Background: Recently, applications of albumin nanoparticles as drug delivery carriers have increased. Most toxicology studies have shown that surface chemistry and size of nanoparticles play an important role in biocompatibility and toxicity.

Objectives: The effect of desolvating agents with different chemical properties on the size of synthesized HSA NPs was investigated.

Materials and Methods: Acetone, ethanol, methanol, and acetonitrile were used to synthesize HSA NPs with controllable size by desolvation method. Scanning electron microscopy (SEM), dynamic light scattering (DLS), and circular dichroism (CD) were employed to characterize produced particles. Finally, the toxicity of HSA NPs synthesized under different conditions was evaluated on PC-12 cells.

Results: The sizes of synthesized particles differed according to the different solvents used. The sizes were $275.3 \mathrm{~nm}$, $155.3 \mathrm{~nm}, 100.11 \mathrm{~nm}$, and $66.2 \mathrm{~nm}$ for acetonitrile, ethanol, acetone, and methanol, respectively. CD showed that larger NPs had more changes in the secondary structures. Finally, the toxicity monitored on the cultured PC-12 cells showed no significant toxic effect through treating with these NPs at different concentrations $\left(0-500 \mu \mathrm{g} \cdot \mathrm{mL}^{-1}\right)$.

Conclusions: The size of HSA NPs has a strong dependency on the desolvating agent. The mechanism in which the desolvating agent affects the size of HSA NPs is complex. Various factors such as dielectric constant, polarity, functional groups, and hydrogen bonding of the solvents have the potential to affect the size and structure of HSA NPs. CD analysis suggested that the solvent denaturing capability had a critical effect on the HSA particle size. The stronger denaturing capability of the solvent resulted in the larger HSA particle size.

Keywords: Desolvating agents; Human serum albumin nanoparticles; Physicochemical properties
\end{abstract}

\section{Background}

In recent years, there has been an increasing attention to the application of nanoparticles (NPs) in drug delivery. The interaction of nanoparticles with cells and biomolecules, and their biocompatibility are modulated by physicochemical properties of NPs including their size, shape, and surface charge $(1,2)$. The size of NPs is an important physical parameter with complex effects on toxicity of NPs.

Human serum albumin (HSA) NPs have gained considerable attention as drug delivery carriers, due to their advantages such as being non-toxic, biodegrad- able, and being stable during storage (3-5). On the other hand, due to their high content of amine and carboxyl groups, HSA NPs can easily be targeted or coated with different ligands or polymers (6-8). Due to these unique features, HSA NPs have implemented in varieties of fields including drug delivery (9-11).

Desolvation is the most common method to synthesize albumin NPs, through which HSA NPs are synthesized with the continuous addition of a desolvator such as ethanol to an aqueous solution of albumin under constant stirring. During the addition of the solvent, the water solubility of albumin reduces. Finally, the 
addition of glutaraldehyde results in particle crosslinking. Various studies have been reported on the effect of different process parameters such as $\mathrm{pH}(4)$, temperature (12), amount of glutaraldehyde (12), stirring rate (13), type of buffer, desolvating agent $(4,13)$, and protein concentration $(4,13)$ on the size and size distribution of HSA NPs.

Desolvating agent is an effective parameter with regard to the size of HSA NPs. Strop et al. (14) studied the effect of solvents with different dielectric constants on the size of HSA NPs. They showed that increasing the concentration of water miscible solvents led to a reduced protein hydration. In addition, decreasing dielectric constant of the aqueous mixture increased the size of the NPs. In their study, it was concluded that desolvating agents with higher dielectric constant led to a smaller particle size.

\section{Objectives}

The novel approach of the present study is to investigate the effect of different desolvating agents on the size and structure of HSA NPs, synthesized by desolvation method. To the best of our knowledge, such a comprehensive study of the effect of desolvating agents on the protein nanoparticles size and structure has not been reported before. To this end, ethanol, acetone, acetonitrile, and methanol were selected as desolvating agents. The desolvating agents have different dielectric constants, functional groups, polarity, and hydrogen bonding potentials. The toxic effects of HSA NPs with different sizes, synthesized using various desolvating agents on PC-12 cells, were evaluated. PC-12 cell is a neuroendocrine cell line with the capability to produce neurotransmitter dopamine (DA), and contains functional DA metabolism pathways (15).

\section{Materials and Methods}

\subsection{Materials}

Human serum albumin (HSA, fraction V), glutaraldehyde ( $8 \%$ aqueous solution), trypsin solution, and 3-[4, 5-dimethylthiazol-2-yl] -2, 5-diphenyltetrazolium bromide (MTT) were obtained from SigmaAldrich. Ethanol, acetone, methanol, and acetonitrile were purchased from Merck. Penicillin-streptomycin, fetal bovine serum (FBS), and Dulbecco's Modified Eagle Medium (DMEM) were purchased from Gibco BRL (Gaithersberg, MD, USA)

\subsection{Preparation of HSA NPS}

Desolvation technique was used for the preparation of HSA NPs (4). Methanol, acetone, ethanol, and acetonitrile were used as desolvating agents. In principle, $100 \mathrm{mg}$ of HSA was dissolved in $1.0 \mathrm{~mL}$ of $10 \mathrm{mM}$ $\mathrm{NaCl}$ solution and $\mathrm{pH}$ was adjusted to 8.2. NPs were synthesized by dropwise addition of desolvating agents at flow rate of $1.0 \mathrm{~mL} \cdot \mathrm{min}^{-1}$ with continuous stirring $(550 \mathrm{rpm})$ at room temperature until the solution became turbid. Subsequently, $60 \mu \mathrm{L}$ of $8 \%$ glutaraldehyde solution was added slowly to induce particle crosslinking, and the solution was stirred for additional $12 \mathrm{~h}$. The resulting NPs were purified by three cycles of centrifugation $(28,000 \times \mathrm{g}, 25 \mathrm{~min})$.

\subsection{Physicochemical Characterization of Nanoparticles}

Particle size, morphology, and structure were studied using MIRA|\TESCAN scanning electron microscopy (SEM). Zeta potential measurements were carried out using a Malvern Zetasizer Nano (S90, UK). Circular dichroism (CD) spectra of HSA NPs were recorded with an Aviv Model-215.

\subsection{Cell Viability Assay}

In vitro cytotoxicity experiments were assessed using MTT reduction. PC-12 cells were seeded in 96well plates for $24 \mathrm{~h}$ at $37^{\circ} \mathrm{C}$. The cells were subcultured in media containing different concentrations of HSA NPs $\left(10-500 \mu \mathrm{g} \cdot \mathrm{mL}^{-1}\right)$. Again, after $24 \mathrm{~h}$, the medium was replaced with the fresh medium containing $10 \%$ MTT (5 mg.mL $\left.{ }^{-1}\right)$ and the plates were incubated for another $4 \mathrm{~h}$ at $37^{\circ} \mathrm{C}$ for the cytotoxicity assay. The medium was removed, and $100 \mu \mathrm{L}$ DMSO was added to each well (16). Absorption values at $570 \mathrm{~nm}$ were determined and cell viability was calculated as follows:

Cell viability $(\%)=\frac{A b s_{570}(\text { Treated cells })}{A b s_{570}(\text { Control cells })} \times 100$

\subsection{Solvent Properties}

The dielectric constant, dipole moment, and solubility parameters of the desolvating agents are listed (Table 1). Total (or Hildebrand) solubility parameter is the sum of the squares of the Hansen solubility parameters (HSP). These parameters arise from (atomic) dispersion forces, (molecular) permanent dipole-permanent dipole forces, and (molecular) hydrogen bonding (electron exchange) (17). Materials with similar HSP have high affinity for each other. The extent of the similarity in a given situation determines the extent of the interaction.

Water, ethanol, and methanol are polar protic sol- 
Table 1. Solubility parameters of desolvating agents

\begin{tabular}{lcccccc}
\hline Solvent & $\begin{array}{c}\text { Dielectric } \\
\text { constant }(\mu)\end{array}$ & $\begin{array}{c}\text { Dipole } \\
\text { moment }\end{array}$ & $\boldsymbol{\delta}_{\boldsymbol{H}^{a}}$ & $\boldsymbol{\delta}_{\boldsymbol{P}^{b}}$ & $\boldsymbol{\delta}_{\boldsymbol{D}^{c}}$ & $\boldsymbol{\delta}^{\boldsymbol{d}}$ \\
\hline acetone & 21 & 2.88 & 7 & 10.4 & 15.5 & 19.9 \\
ethanol & 24.5 & 1.69 & 19.4 & 8.8 & 15.8 & 26.2 \\
methanol & 33 & 1.7 & 22.3 & 12.3 & 14.7 & 26.9 \\
acetonitrile & 37.5 & 3.94 & 6.1 & 18 & 15.3 & 24.3 \\
water & 88 & 1.84 & 42.3 & 16 & 15.5 & 48 \\
\hline
\end{tabular}

ahydrogen bonding solubility parameter ( $\left.\mathrm{Mpa}^{0.5}\right)$

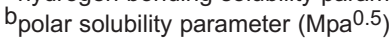

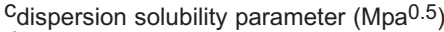

$\mathrm{d}_{\text {total }}\left(\right.$ Hildebrand) solubility parameter $\left(\mathrm{Mpa}^{0.5}\right)\left(\delta^{2}=\delta^{2} D^{+} \delta^{2} H^{+} \delta^{2} P\right)$

vents, which possess $\mathrm{O}-\mathrm{H}$ bonds and can participate in hydrogen bonding. Meanwhile, acetone and acetonitrile are polar aprotic solvents and do not participate in hydrogen bonding.

Using the mixture equation of Silberstein (18), the dielectric constants of an aqueous solution can be determined from the dielectric constants of the pure components and their relative volume:

$\varepsilon=\varepsilon_{1} \bar{v}_{1}+\varepsilon_{2} \bar{v}_{2}$

where $\varepsilon$ is the dielectric constant of mixture, anddenote dielectric constant of each component, and $\bar{v}_{1}$ and $\bar{v}_{2}$ are their relative volume, respectively.

\section{Results}

\subsection{Effect of Desolvating Agents on Physicochemical Properties of HSA NPS}

The amounts of added solvents are shown in Figure 1. Solvents were added to the protein solution until the solution became just turbid. Further addition of desolvating agent led to an increase in the particle density. The results

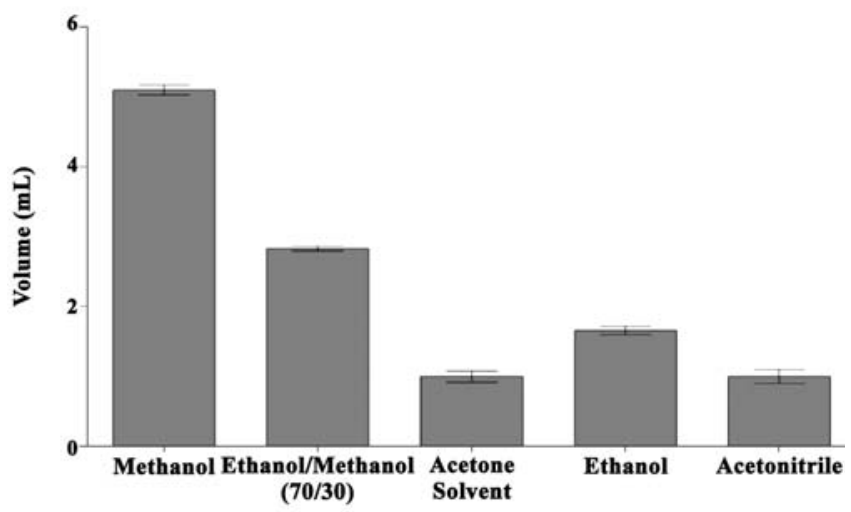

Figure 1. The volume of each desolvating agent added to $1 \mathrm{~mL}$ protein solution until it becomes turbid (mean $\pm \mathrm{SD}, \mathrm{n}=3$ ) showed that all the desolvating agents were able to achieve nanoparticles albumin desolvation.

The size and size distribution of HSA NPs synthesized using different desolvating agents are presented (Table 2). Increasing the ratio of methanol in the mixture of methanol/ethanol (from 0 to 1 ) led to a reduction in the size of HSA NPs (from $155.3 \pm 4.3 \mathrm{~nm}$ to $66.2 \pm 5.5 \mathrm{~nm}$ ), while the polydispersity of the NPs remained almost the same. Also, the amount of added solvents to create turbidity in the protein solution increased with increasing the concentration of methanol. Instead of intense turbidity, they showed slight opalescence, which could be due to the smaller size of HSA NPs synthesized by methanol.

Acetone and acetonitrile are polar aprotic solvents, which have respectively smaller and larger dielectric constants compared to ethanol. The use of acetone led to the synthesis of the NPs with the diameter of $100.11 \pm 4.6 \mathrm{~nm}$, which were smaller than those synthesized using ethanol $(155.3 \pm 4.3 \mathrm{~nm})$. The amount of acetone needed to create turbidity in the protein solution was lower than ethanol $(1 \pm 0.08 \mathrm{~mL}$ vs $1.66 \pm 0.06$ $\mathrm{mL}$ ). Despite the smaller dielectric constant of acetone compared to ethanol, the dielectric constant of the acetone-protein mixture was higher (51.5 versus 45.49 ), due to the lower final concentration of acetone in the solution.

Among the desolvating agents, acetonitrile with the largest dielectric constant was used to synthesize HSA NPs. Based on its dielectric constant, it was anticipated that acetonitrile would lead to the production of the smallest HSA NPs. But the results showed that HSA NPs obtained by the use of acetonitrile had both the largest size $(275.3 \pm 15 \mathrm{~nm})$ and size distribution $(0.45 \pm 0.05 \mathrm{~nm})$. By the way, the amount of acetonitrile needed to create turbidity in the protein solution was $1 \pm 0.1 \mathrm{~mL}$.

Figure 2 shows the CD spectra of HSA NPs synthesized using different desolvating agents. The deconvo-

Table 2. Size and size distribution of HAS NPs synthesized using different desolvating agents

\begin{tabular}{lccc}
\hline Solvent & $\begin{array}{c}\text { Functional } \\
\text { group }\end{array}$ & Size $(\mathbf{n m})$ & Pdl \\
\hline methanol & $-\mathrm{OH}$ & $66.2 \pm 5.5$ & $0.047 \pm 0.007$ \\
methanol/etha & $-\mathrm{OH}$ & $78.62 \pm 4.2$ & $0.03 \pm 0.005$ \\
nol $(30 / 70)$ & & & \\
acetone & $-\mathrm{C}=\mathrm{O}$ & $100.11 \pm 4.6$ & $0.036 \pm 0.009$ \\
ethanol & $-\mathrm{OH}$ & $155.3 \pm 4.3$ & $0.038 \pm 0.01$ \\
acetonitrile & $\mathrm{C} \equiv \mathrm{N}$ & $275.3 \pm 15$ & $0.45 \pm 0.05$ \\
\hline
\end{tabular}




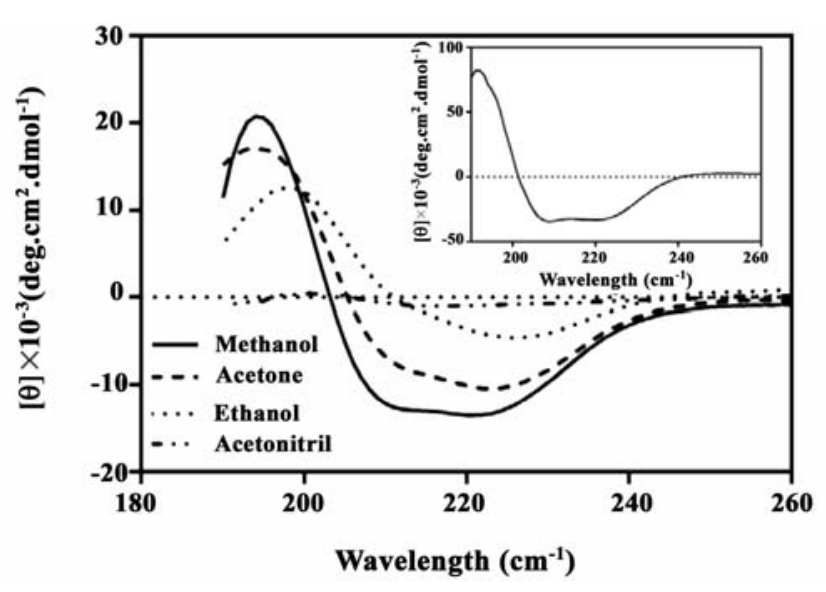

Figure 2. CD spectra of HAS NPs synthesized using different desolvating agents. Inset: CD spectra of HAS protein. CD analysis showed that larger NPs had more changes in the secondary structures

lution of CD spectra of HSA NPs showed that the use of desolvating agents led to a major reduction of protein $\alpha$-helical structure. HSA NPs produced by methanol were smaller than particles produced by acetonitrile. Moreover, $\alpha$-helical structure was reduced just a little as opposed to the time that acetonitrile was used.

The SEM images of HSA NPs are illustrative of spherical HSA NPs with a smooth surface morphology (Figure 3). However, the NPs synthesized using acetonitrile produced larger particle sizes, being more polydisperse than the others (Figure 3D).

The type of desolvating agent had no significant effect on the zeta potential of HSA NPs (Table 3). The zeta potentials of the NPs were independent of the desolvating agent, ranging between -33 and $-38 \mathrm{mV}$.

\subsection{Cytotoxicity of HSA NPS}

In order to evaluate the cytotoxic effect of different particle preparations on $\mathrm{PC}-12$ cells and the toxicity of the different sizes of HSA NPs, the in vitro acute cytotoxicity was measured using MTT assay. PC-12 cells

Table 3. Zeta potential of NPs synthesized by different desolvating agents

\begin{tabular}{lc}
\hline Solvent & Zeta potential $(\mathbf{m V})$ \\
\hline methanol & $-35 \pm 2.2$ \\
ethanol/methanol $(70 / 30)$ & $-34 \pm 2.2$ \\
acetone & $-33 \pm 3.1$ \\
ethanol & $-38 \pm 2.9$ \\
acetonitrile & $-33 \pm 2.3$ \\
\hline
\end{tabular}
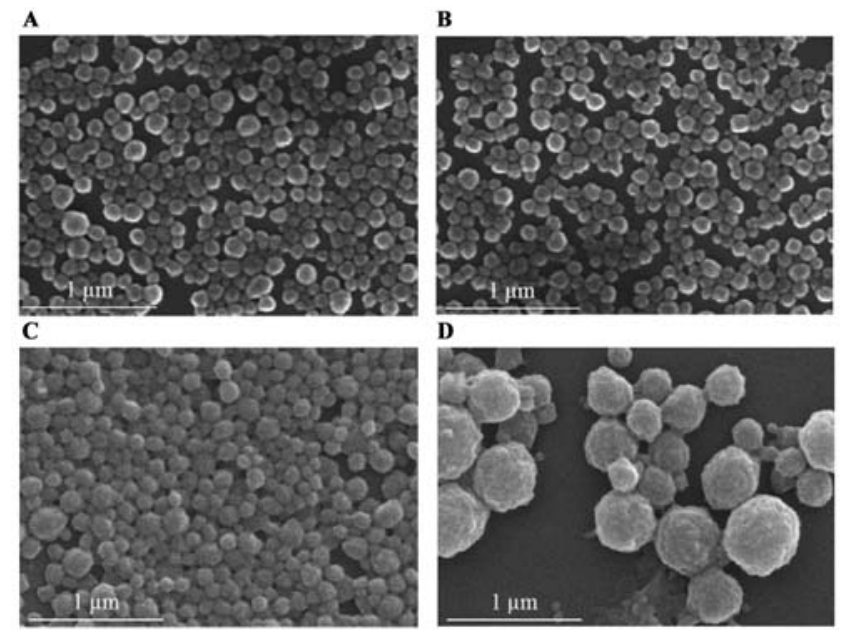

Figure 3. NPs synthesized using different desolvating agents: A: methanol, B: acetone, C: ethanol, D: acetonitrile

were incubated with different concentrations of HSA NPs $\left(0-500 \mu \mathrm{g} \cdot \mathrm{mL}^{-1}\right)$. The results showed no significant toxicity of HSA NPs at different concentrations (data not shown).

\section{Discussion}

The main goal of this work was to study the effect of desolvating agents with different dielectric constants, functional groups, polarities, and hydrogen bonding potentials on the size and polydispersity of HSA-NP. Strop et al. (14) who worked on the effect of dielectric constant of desolvating agents on the size of HSA NPs found a correlation between the dielectric constant and resulting particle size in the desolvation procedure. It was assumed that desolvating agents with higher dielectric constants lead to smaller particle size. However, our data was suggestive of inconsistencies in previously presented correlation

HSA is a compactly folded protein, including the interior hydrophobic region and hydrophilic layer on the surface of the molecule (19). By considering the three dimensional structure of protein, various phenomena regarding the changes in the structure of HSA can be explained as described below.

HSA protein in water is mostly hydrated and a hydrophilic shell surrounds the protein. The denaturing ability of a water-miscible desolvating agent is dependent on its ability to enter into the hydrophobic region by disrupting the outside hydration layer. Consequently, both hydrophobic and hydrophilic potential of desolvating agents can simultaneously affect the amount of denaturation of the protein and additionally the size of NPs. 
When a mixture of polar protic solvents such as methanol and ethanol was used as desolvating agent, the size of HSA NPs decreased with increasing the concentration of methanol, which is in accordance with the results of Strop et al. (14). On the other hand, the amount of added solvent to make protein solution turbid increased with an increase in methanol concentration, which is due to the less ability of methanol, compared with ethanol, to precipitate albumin in the aqueous solution. Previously, June et al. (20) showed that the end point for HSA particle size control was not sensitive. In other words, increased turbidity indicates an increase of density within particles and not in size. Methanol can easily disrupt the outer hydrophilic layer of protein. According to (Table 1), the $\delta_{p}$ and $\delta_{H}$ of methanol are more than those of ethanol and its $\delta$ value is nearer to the $\delta$ value of water. However, ethanol is more effective than methanol in solubilizing nonpolar groups (21), since ethanol disrupts the protein structure with lesser volumes. Furthermore, CD data indicated that ethanol led to extreme changes in the content of secondary structure of albumin, supporting its efficiency.

Use of acetone, a desolvating agent with smaller value of dielectric constant than ethanol, led to the synthesis of HSA NPs with smaller sizes than those synthesized using ethanol. The ability of ethanol to disrupt the outer layer of protein is more than acetone (based on its $\delta_{H}$ and $\delta_{H}$ ); however, acetone has more ability to interact with non-polar regions of proteins. The results of CD spectra showed that the changes in the structure of albumin by acetone were less than the changes induced by ethanol. It caused less protein aggregation and consequently smaller sizes of NPs. Meanwhile, the dielectric constant of solution calculated by the Silberstein equation showed that it was higher when acetone was used (51.5 vs 45.49), which is due to the less required amount of acetone to make the solution turbid. Higher dielectric constant and less change in the structure of albumin synthesized by acetone compared with those synthesized by ethanol can affect the interfacial tension and the free enthalpy of particles. This in turn allows the increase of surface to volume ratio that leads to smaller NPs. On the other hand, the presence of hydrogen bonds facilitates the formation of larger lattices and consequently larger particles. Acetone acts as a hydrogen bond acceptor, while ethanol is polar protic solvent that can act as both donor and acceptor of hydrogen bond. Less formation of hydrogen bonds by acetone enables the production of smaller HSA NPs than those synthesized by ethanol. Azarmi et al. (22) studied the effect of acetone and ethanol on the size of gelatin NPs. They showed that the NPs were synthesized with acetone were generally smaller and had a lower polydispersity index compared to those prepared with ethanol.

Acetonitrile as another polar aprotic solvent was used to synthesis HSA NPs. Acetonitrile can induce denaturation in the protein structure by altering the tertiary structure through hydrophobic interaction (23). Introduction of acetonitrile into the aqueous protein solution increases the chemical potential of the protein, which causes the system to become thermodynamically destabilized. Since it has the highest value of dielectric constant, it was anticipated to obtain HSA NPs of the smallest sizes. However, the results showed that HSA NPs with the size of $275.3 \pm 15$ were prepared using acetonitrile with the lowest amount of solvent required to make the solution turbid. $\delta_{H}$ value of acetonitrile was smaller than the $\delta_{H}$ value of other solvents. On the other hand, Gekko et al. (23) showed that acetonitrile has high tendency to interact with nonpolar side chains, which leads to denaturation of protein and an increase in the chemical state of protein. Moreover, the results of CD spectra showed that acetonitrile as a desolvating agent leads to the highest changes in the structure of HSA protein, compared with other desolvating agents. Altogether, due to its high tendency to interact with nonpolar groups and denature albumin, acetonitrile leads to the highest rate of preparation of large aggregates of HSA NPs, and consequently results in the preparation of the NPs with high polydispersity index (PDI) value.

In summary, it was found that except dielectric constant that was stated in the study by Von strop et al. (14), other structural and solubility parameters of desolvating agents can affect the size and polydispersity of protein NPs, and it is an oversimplification to assume that using desolvating agents with higher dielectric constants leads to smaller particle size.

In order to assess the toxic effect of HSA NPs with different sizes prepared, using different desolvating agents, PC-12 cells were incubated with the NPs. It has been previously shown that the desolvating agents have toxicity on cells (14). As no significant toxicity of the NPs was observed, except at high concentration of those synthesized by methanol, it can be concluded that the HSA NPs of different sizes (65 to $275 \mathrm{~nm}$ ) have no toxicity and the method of eliminating solvent and unreacted glutaraldehyde is effective, which shows that these NPs are biocompatible. 


\section{References}

1. Kang T, Guan R, Chen X, Song Y, Jiang H, Zhao J. In vitro toxicity of different-sized $\mathrm{ZnO}$ nanoparticles in Caco-2 cells. Nanoscale Res Lett. 2013;8(1):1-8. DOI: 10.1186/1556-276X$8-496$

2. Álvarez YD, Fauerbach JA, Pellegrotti JV, Jovin TM, JaresErijman EA, Stefani FD. Influence of Gold nanoparticles on the kinetics of $\alpha$-synuclein aggregation. Nano lett. 2013;13(12):6156-6163. DOI: 10.1021/nl403490e

3. Elzoghby AO, Samy WM, Elgindy NA. Albumin-based nanoparticles as potential controlled release drug delivery systems. J Controlled Release. 2012;157(2):168-182.

4. Langer K, Balthasar S, Vogel V, Dinauer N, Von Briesen H, Schubert D. Optimization of the preparation process for human serum albumin (HSA) nanoparticles. Int $J$ Pharm. 2003;257(1):169-180. DOI: 10.1016/S0378-5173(03)00134-0

5. Jahanshahi M. Re-design of downstream processing techniques for nanoparticulate bioproducts. Iran J Biotech. 2004;2(1):1-12.

6. Kouchakzadeh H, Shojaosadati SA, Tahmasebi F, Shokri F. Optimization of an anti-HER2 monoclonal antibody targeted delivery system using PEGylated human serum albumin nanoparticles. Int $J$ Pharm. 2013;447(1):62-69. DOI: 10.1016/j.ijpharm.2013.02.043

7. Patel PJ, Acharya NS, Acharya SR. Development and characterization of glutathione-conjugated albumin nanoparticles for improved brain delivery of hydrophilic fluorescent marker. Drug Deliv. 2013;20(3-4):143-155. DOI: 10.3109/107175 44.2013.801050

8. Kouchakzadeh H, Shojaosadati SA, Maghsoudi A, Farahani EV. Optimization of PEGylation conditions for BSA nanoparticles using response surface methodology. AAPS Pharm Sci Tech. 2010;11(3):1206-1211. DOI: 10.1208/s12249-010-948 7-8

9. Luppi B, Bigucci F, Corace G, Delucca A, Cerchiara T, Sorrenti $\mathrm{M}$, et al. Albumin nanoparticles carrying cyclodextrins for nasal delivery of the anti-Alzheimer drug tacrine. Eur J Pharm Sci. 2011;44(4):559-565. DOI: 10.1016/j.ejps.2011.10.002

10. Zu Y, Zhang Y, Zhao X, Zhang Q, Liu Y, Jiang R. Optimization of the preparation process of vinblastine sulfate (VBLS)-loaded folateconjugated bovine serum albumin (BSA) nanoparticles for tumor-targeted drug delivery using response surface methodology (RSM). Int J Nanomed. 2009;4:321. DOI: http://dx.doi.org/10.2147/IJN.S8501

11. Merodio M, Arnedo A, Renedo MJ, Irache JM. Ganciclovirloaded albumin nanoparticles: characterization and in vitro release properties. Eur J Pharm Sci. 2001;12(3):251-259. DOI: 10.1016/S0928-0987(00)00169-X

12. Weber C, Kreuter J, Langer K. Desolvation process and surface characteristics of HSA-nanoparticles. Int $J$ Pharm. 2000;196(2):197-200. DOI: 10.1016/S0378-5173 (99) 00420-2

13. Jun JY, Nguyen HH, Paik S-Y-R, Chun HS, Kang B-C, Ko S. Preparation of size-controlled bovine serum albumin (BSA) nanoparticles by a modified desolvation method. Food Chem. 2011;127(4):1892-1898. DOI: 10.1016/j.foodchem.2011.02. 040

14. Von Storp B, Engel A, Boeker A, Ploeger M, Langer K. Albumin nanoparticles with predictable size by desolvation procedure. J Microencapsulation. 2012;29(2):138-146. DOI:
10.3109/02652048.2011.635218

15. Hussain SM, Javorina AK, Schrand AM, Duhart HM, Ali SF, Schlager JJ. The interaction of manganese nanoparticles with PC-12 cells induces dopamine depletion. TToxicol Sci. 2006;92(2):456-463.

16. Hansen MB, Nielsen SE, Berg K. Re-examination and further development of a precise and rapid dye method for measuring cell growth/cell kill. J Immunol Methods. 1989;119(2):203210. DOI: 10.1016/0022-1759(89)90397-9

17. Hansen CM. Hansen solubility parameters: a user's handbook. CRC press. 2012. DOI: 10.1201/9781420049312.ch1

18. Arnold K, Herrmann A, Pratsch L, Gawrisch K. The dielectric properties of aqueous solutions of poly (ethylene glycol) and their influence on membrane structure. BBA Biomemb. 1985;815(3):515-518. DOI: 10.1016/0005-2736(85)90381-5

19. Fukushima D. Denaturation of soybean proteins by organic solvents. Cereal Chem. 1969;46 (2):156.

20. Nguyen HH, Ryu J, Che JH, Kang TS, Lee JK, Song CW, et al. Robust size control of bovine serum albumin (BSA) nanoparticles by intermittent addition of a desolvating agent and the particle formation mechanism. Food Chem. 2013;141(2):695701. DOI: $10.1016 /$ j.foodchem.2013.04.059

21. Nozaki Y, Tanford C. The solubility of amino acids and two glycine peptides in aqueous ethanol and dioxane solutions establishment of a hydrophobicity scale. J Biol Chem. 1971;246(7):2211-2217.

22. Azarmi S, Huang Y, Chen H, McQuarrie S, Abrams D, Roa W, et al. Optimization of a two-step desolvation method for preparing gelatin nanoparticles and cell uptake studies in 143B osteosarcoma cancer cells. J Pharm Pharm Sci. 2006;9(1):124-132.

23. Gekko K, Ohmae E, Kameyama K, Takagi T. Acetonitrileprotein interactions: amino acid solubility and preferential solvation. BBA Protein Struct M. 1998;1387(1):195-205. DOI: $10.1016 / \mathrm{S} 0167-4838(98) 00121-6$ 